\title{
Open charm-bottom axial-vector tetraquarks and their properties
}

\author{
S. S. $\operatorname{Agaev}^{1}$, K. Azizi ${ }^{2, a}$, H. Sundu ${ }^{3}$ \\ ${ }^{1}$ Institute for Physical Problems, Baku State University, Az 1148 Baku, Azerbaijan \\ 2 Department of Physics, Doğuş University, Acibadem-Kadiköy, 34722 Istanbul, Turkey \\ ${ }^{3}$ Department of Physics, Kocaeli University, 41380 Izmit, Turkey
}

Received: 12 March 2017 / Accepted: 5 May 2017 / Published online: 18 May 2017

(C) The Author(s) 2017. This article is an open access publication

\begin{abstract}
The charged axial-vector $J^{P}=1^{+}$tetraquarks $Z_{q}=[c q][\bar{b} \bar{q}]$ and $Z_{s}=[c s][\bar{b} \bar{s}]$ with the open charmbottom contents are studied in the diquark-antidiquark model. The masses and meson-current couplings of these states are calculated by employing a QCD two-point sum rule approach, where the quark, gluon and mixed condensates up to eight dimensions are taken into account. These parameters of the tetraquark states $Z_{q}$ and $Z_{s}$ are used to analyze the vertices $Z_{q} B_{c} \rho$ and $Z_{s} B_{c} \phi$ to determine the strong $g_{Z_{q} B_{c} \rho}$ and $g_{Z_{s} B_{c} \phi}$ couplings. For these purposes, the QCD light-cone sum rule method and its soft-meson approximation are utilized. The couplings $g_{Z_{q} B_{c} \rho}$ and $g_{Z_{s} B_{c} \phi}$, extracted from this analysis, are applied for evaluating the strong $Z_{q} \rightarrow B_{c} \rho$ and $Z_{s} \rightarrow B_{c} \phi$ decays' widths, which are essential results of the present investigation. Our predictions for the masses of the $Z_{q}$ and $Z_{s}$ states are confronted with similar results available in the literature.
\end{abstract}

\section{Introduction}

Charmonium-like states discovered during the last years mainly in the exclusive B-meson decays as resonances in the relevant mass distributions became interesting objects for both experimental and theoretical studies in high energy physics. Conventional hadrons, composed of two and three quarks, and investigated in a rather detailed form, constitute the main part of the known particles. At the same time, the theory of the strong interactions-Quantum Chromodynamics - does not contain principles excluding the existence of the multi-quark states. The tetraquark and pentaquark states composed of four and five valence quarks, respectively, and hybrids built by quarks and gluons are among the most promising candidates to occupy the vacant shelves in the multi-quark spectroscopy. Due to joint efforts of experimentalists and theorists considerable progress in

a e-mail: azizi.hep.ph@gmail.com understanding of the quark-gluon structure of the multiquark-exotic states and explaining their properties-was achieved, but remaining questions are more numerous than answered ones (for the latest reviews, see Refs. [1-4]).

The main source of problems, which complicates the study of the charmonium-like tetraquarks, is the existence of conventional charmonium states in the energy ranges of the decay processes to be explored. Charmonia generates difficulties in the interpretation of experimental results, because the pure $c \bar{c}$ states may emerge as the resonances in the mass distributions of the processes, or generate background effects due to states dynamically connected with $c \bar{c}$ levels. Only after eliminating effects of the charmonium states in forming of the experimental data, the observed resonances can be considered as real exotic particles. The well-known $X(3872)$ state is the best sample to illustrate existing problems. It was discovered as a very narrow resonance in B-meson decay $B \rightarrow K X \rightarrow K J / \psi \rho \rightarrow K J / \psi \pi^{+} \pi^{-}$by the Belle Collaboration [5], and it was later confirmed in CDF, D0 and BaBar experiments (see Refs. [6-8]). Its other production mechanisms running through decay chains $B \rightarrow K X \rightarrow$ $K J / \psi \omega \rightarrow K J / \psi \pi^{+} \pi^{-} \pi^{0}, B \rightarrow K X \rightarrow K J / \psi \gamma$ and $B \rightarrow K X \rightarrow K \psi(2 S) \gamma$ were also experimentally measured and comprehensively studied $[9,10]$. The gathered information poses severe restrictions on theoretical models claiming to describe a behavior of the $X(3872)$ state. Attempts were made to explain the collected data by treating $X(3872)$ as the excited conventional charmonium $\chi_{c 1}\left(2^{3} P_{1}\right)$ [11], or as the state formed due to dynamical coupled-channel effects [12]. It was considered in the context of four-quark compounds, both as the $D \bar{D}^{\star}$ molecule or its admixtures with the charmonium states [13-16], and as the diquark-antidiquarks states [17-21].

But tetraquarks, which do not contain $\bar{c} c$ or $\bar{b} b$ pairs might also exist, because the fundamental laws of QCD do not forbid production of such resonances in hadronic processes. These particles may appear in the exclusive reactions 
as the open charm (i.e., as states containing $c$ or $\bar{c}$ quarks) and open bottom resonances. The $D_{s 0}^{\star}(2317)$ and $D_{s 1}(2460)$ mesons, discovered by the BaBar and CLEO Collaborations $[22,23]$, are now being considered as candidates for open charm tetraquark states. The $X(5568)$ resonance remains a unique candidate for the open bottom tetraquark, which is also a particle containing four different quarks. Unfortunately, the experimental situation formed around $X(5568)$ remains unclear. Indeed, the evidence for $X(5568)$ was first announced by the D0 Collaboration in Ref. [24]. Later it was seen again by D0 in the $B_{s}^{0}$ meson's semileptonic decays [25]. Nevertheless, the LHCb and CMS Collaborations could not see the same resonance by an analysis of their experimental data $[26,27]$. Theoretical investigations aiming to explain the nature of $X(5568)$ and calculate its parameters lead also to contradictory conclusions. Predictions obtained in some of this work are in a nice agreement with results of the D0 Collaboration, while in others even the existence of the $X(5568)$ state is an object of doubt. The detailed discussions of these and related questions of the physics of the $X$ (5568) state can be found in the original work (see Ref. [2] and the references therein).

The open charm-bottom tetraquarks belong to another type of exotic states. They already attracted the interest of physicists even though still they have not been observed experimentally. The original investigations of these particles started more than two decades ago, and, therefore, a considerable theoretical information on their expecting properties is available in the literature. For example, the open charm-bottom type tetraquarks with the contents $\{Q q\}\left\{Q^{\prime} q\right\}$, $\{Q s\}\left\{Q^{\prime} s\right\}$ and molecule structures were considered in Refs. $[28,29]$, respectively. In these papers the masses of these hypothetical states were calculated in the context of the QCD two-point sum rule approach using in the operator product expansion (OPE) operators up to dimension six. In the framework of the diquark-antidiquark model the open charmbottom states were analyzed in Ref. [30]. In order to extract masses of these states, the authors again utilized the QCD sum rule method, interpolating currents of different color structure. Other aspects of these tetraquark systems can be found in Refs. [31-35].

In a previous article [36] we explored the charged scalar $J^{P}=0^{+}$tetraquark states $Z_{q}=[c q][\bar{b} \bar{q}]$ and $Z_{s}=[c s][\bar{b} \bar{s}]$ in the context of the diquark-antidiquark model, and we calculated their masses and the widths of some of their decay channels. In the present work we extend our investigations by including into analysis the axial-vector $J^{P}=1^{+}$ $Z_{q}=[c q][\bar{b} \bar{q}]$ and $Z_{s}=[c s][\bar{b} \bar{s}]$ open charm-bottom tetraquarks, and their kinematically allowed decay modes.

We start from calculation of their masses and mesoncurrent couplings. For these purposes, we employ the QCD two-point sum rule method, which was invented to calculate the parameters of the conventional hadrons [37], but it soon was applied to an analysis of the exotic states as well (see Refs. [38-43]). The parameters of the open charm-bottom tetraquarks obtained within this method are used to explore the strong vertices $Z_{q} B_{c} \rho$ and $Z_{s} B_{c} \phi$ and calculate the corresponding couplings $g_{Z_{q} B_{c} \rho}$ and $g_{Z_{s} B_{c} \phi}$. These couplings are required to evaluate the widths of the $Z_{q} \rightarrow B_{c} \rho$ and $Z_{s} \rightarrow B_{c} \phi$ decays. To this end, we apply the QCD lightcone sum method and the soft-meson approximation proposed in Refs. [44-46]. For analysis of the strong vertices of tetraquarks the method was, for the first time, examined in Ref. [47], and afterwards successfully used to investigate decay channels of some tetraquarks states (see Refs. [4850]).

The present work is organized in the following manner. In Sect. 2 we calculate the masses and meson-current couplings of the axial-vector tetraquarks with open charm-bottom contents. Section 3 is devoted to the computation of the strong couplings $g_{Z_{q} B_{c} \rho}$ and $g_{Z_{s} B_{c} \phi}$. In this section we calculate the widths of the decays $Z_{q} \rightarrow B_{c} \rho$ and $Z_{s} \rightarrow B_{c} \phi$. In Sect. 4 we examine our results as part of the general tetraquark's physics and compare them with predictions of Ref. [30], where the masses of the axial-vector open charmbottom tetraquarks were found. It contains also our concluding remarks.

\section{Masses and meson-current couplings}

In order to find the masses and meson-current couplings of the diquark-antidiquark type axial-vector states $Z_{q}$ and $Z_{s}$, we use the two-point QCD sum rules. Below the explicit expressions for the $Z_{q}$ state are written down. Their generalization to the $Z_{s}$ tetraquark is straightforward.

The two-point sum rule can be extracted from an analysis of the correlation function

$\Pi_{\mu \nu}(p)=i \int \mathrm{d}^{4} x e^{i p x}\left\langle 0\left|\mathcal{T}\left\{J_{\mu}(x) J_{v}^{\dagger}(0)\right\}\right| 0\right\rangle$,

where $J_{\mu}$ is the interpolating current of the $Z_{q}$ state.

The scalar and axial-vector open charm-bottom diquarkantidiquark states can be modeled using different type of interpolating currents [30]. Thus, the interpolating currents can be either symmetric or antisymmetric in the color indices. In our previous work we chose the symmetric interpolating current to find masses and decay widths of the scalar open charm-bottom tetraquarks [36]. In the present work to consider the axial-vector tetraquark states $Z_{q}$ and $Z_{s}$ we use again the interpolating currents, which are symmetric in the color indices. Such an axial-vector current has the following form:

$J_{\mu}=q_{a}^{T} C \gamma_{5} c_{b}\left(\bar{q}_{a} \gamma_{\mu} C \bar{b}_{b}^{T}+\bar{q}_{b} \gamma_{\mu} C \bar{b}_{a}^{T}\right)$, 
and it is symmetric under exchange of the color indices $a \leftrightarrow b$. Here by $C$ we denote the charge conjugation matrix.

To derive QCD sum rules for the mass and meson-current coupling we follow standard prescriptions of the sum rule method and express the correlation function $\Pi_{\mu \nu}(p)$ in terms of the physical parameters of the $Z_{q}$ state, which results in obtaining $\Pi_{\mu \nu}^{\text {Phys }}(p)$. From another side the same function should be obtained in terms of the quark-gluon degrees of freedom $\Pi_{\mu \nu}^{\mathrm{QCD}}(p)$.

We start from the function $\Pi_{\mu \nu}^{\text {Phys }}(p)$ and compute it by suggesting that the tetraquarks under consideration are the ground states in the relevant hadronic channels. After saturating the correlation function with a complete set of the $Z_{q}$ states and performing in Eq. (1) integration over $x$, we get the required expression for $\Pi_{\mu \nu}^{\text {Phys }}(p)$,

$\Pi_{\mu \nu}^{\text {Phys }}(p)=\frac{\left\langle 0\left|J_{\mu}\right| Z_{q}(p)\right\rangle\left\langle Z_{q}(p)\left|J_{\nu}^{\dagger}\right| 0\right\rangle}{m_{Z}^{2}-p^{2}}+\cdots$

where $m_{Z}$ is the mass of the $Z_{q}$ state, and dots indicate contributions coming from higher resonances and continuum states. We introduce the meson-current coupling $f_{Z}$ by means of the equality

$\left\langle 0\left|J_{\mu}\right| Z_{q}(p)\right\rangle=f_{Z} m_{Z} \varepsilon_{\mu}$,

where $\varepsilon_{\mu}$ is polarization vector of the axial-vector tetraquark. In terms of $m_{Z}$ and $f_{Z}$ the correlation function takes the simple form

$\Pi_{\mu \nu}^{\text {Phys }}(p)=\frac{m_{Z}^{2} f_{Z}^{2}}{m_{Z}^{2}-p^{2}}\left(-g_{\mu \nu}+\frac{p_{\mu} p_{v}}{m_{Z}^{2}}\right)+\cdots$.

Having applied the Borel transformation to the function $\Pi_{\mu \nu}^{\text {Phys }}(p)$ we get

$\mathcal{B}_{p^{2}} \Pi_{\mu \nu}^{\text {Phys }}\left(p^{2}\right)=m_{Z}^{2} f_{Z}^{2} e^{-m_{Z}^{2} / M^{2}}\left(-g_{\mu \nu}+\frac{p_{\mu} p_{v}}{m_{Z}^{2}}\right)+\ldots$

In order to obtain the function $\Pi_{\mu \nu}^{\mathrm{QCD}}(p)$ we substitute the interpolating current given by Eqs. (2) into (1), and we employ the light and heavy quark propagators in calculations. For $\Pi_{\mu \nu}^{\mathrm{QCD}}(p)$, as a result, we get

$$
\begin{aligned}
\Pi_{\mu \nu}^{\mathrm{QCD}}(p)= & i \int \mathrm{d}^{4} x e^{i p x}\left\{\operatorname{Tr}\left[\gamma_{\mu} \widetilde{S}_{b}^{b^{\prime} b}(-x) \gamma_{\nu} S_{q}^{a^{\prime} a}(-x)\right]\right. \\
& \times \operatorname{Tr}\left[\gamma_{5} \widetilde{S}_{q}^{a a^{\prime}}(x) \gamma_{5} S_{c}^{b b^{\prime}}(x)\right]+\operatorname{Tr}\left[\gamma_{\mu} \widetilde{S}_{b}^{a^{\prime} b}(-x)\right. \\
& \left.\times \gamma_{\nu} S_{q}^{b^{\prime} a}(-x)\right] \operatorname{Tr}\left[\gamma_{5} \widetilde{S}_{q}^{a^{\prime}}(x) \gamma_{5} S_{c}^{b b^{\prime}}(x)\right]
\end{aligned}
$$

$$
\begin{aligned}
& +\operatorname{Tr}\left[\gamma_{\mu} \widetilde{S}_{b}^{b^{\prime} a}(-x) \gamma_{\nu} S_{q}^{a^{\prime} b}(-x)\right] \operatorname{Tr}\left[\gamma_{5} \widetilde{S}_{q}^{a a^{\prime}}(x) \gamma_{5} S_{c}^{b b^{\prime}}(x)\right] \\
& \left.+\operatorname{Tr}\left[\gamma_{\mu} \widetilde{S}_{b}^{a^{\prime a} a}(-x) \gamma_{\nu} S_{q}^{b^{\prime} b}(-x)\right] \operatorname{Tr}\left[\gamma_{5} \widetilde{S}_{q}^{a a^{\prime}}(x) \gamma_{5} S_{c}^{b b^{\prime}}(x)\right]\right\},
\end{aligned}
$$

where

$\widetilde{S}_{q(b)}^{a b}(x)=C S_{q(b)}^{T a b}(x) C$,

with $S_{q}(x)$ and $S_{b}(x)$ being the $q$ - and $b$-quark propagators, respectively.

We proceed including into the analysis the well-known expressions of the light and heavy quark propagators. For our aims it is convenient to use the $x$-space expression of the light quark propagator,

$$
\begin{aligned}
S_{q}^{a b}(x)= & i \delta_{a b} \frac{\not}{2 \pi^{2} x^{4}}-\delta_{a b} \frac{m_{q}}{4 \pi^{2} x^{2}}-\delta_{a b} \frac{\langle\bar{q} q\rangle}{12} \\
& +i \delta_{a b} \frac{\not m_{q}\langle\bar{q} q\rangle}{48}-\delta_{a b} \frac{x^{2}}{192}\left\langle\bar{q} g_{s} \sigma G q\right\rangle \\
& +i \delta_{a b} \frac{x^{2} \not m_{q}}{1152}\left\langle\bar{q} g_{s} \sigma G q\right\rangle-i \frac{g_{s} G_{a b}^{\alpha \beta}}{32 \pi^{2} x^{2}}\left[\not \sigma_{\alpha \beta}+\sigma_{\alpha \beta} \not x\right] \\
& -i \delta_{a b} \frac{x^{2} \not g_{s}^{2}\langle\bar{q} q\rangle^{2}}{7776}-\delta_{a b} \frac{x^{4}\langle\bar{q} q\rangle\left\langle g_{s} q^{2} G^{2}\right\rangle}{27648}+\cdots
\end{aligned}
$$

For the heavy $Q=b, c$ quarks we utilize the propagator $S_{Q}^{a b}(x)$ given in the momentum space in Ref. [51]:

$$
\begin{aligned}
& S_{Q}^{a b}(x)=i \int \frac{\mathrm{d}^{4} k}{(2 \pi)^{4}} e^{-i k x}\left\{\frac{\delta_{a b}\left(k+m_{Q}\right)}{k^{2}-m_{Q}^{2}}\right. \\
& -\frac{g_{s} G_{a b}^{\alpha \beta}}{4} \frac{\sigma_{\alpha \beta}\left(k+m_{Q}\right)+\left(k+m_{Q}\right) \sigma_{\alpha \beta}}{\left(k^{2}-m_{Q}^{2}\right)^{2}} \\
& +\frac{g_{s}^{2} G^{2}}{12} \delta_{a b} m_{Q} \frac{k^{2}+m_{Q} k}{\left(k^{2}-m_{Q}^{2}\right)^{4}}+\frac{g_{s}^{3} G^{3}}{48} \delta_{a b} \frac{\left(k+m_{Q}\right)}{\left(k^{2}-m_{Q}^{2}\right)^{6}} \\
& \left.\quad \times\left[k\left(k^{2}-3 m_{Q}^{2}\right)+2 m_{Q}\left(2 k^{2}-m_{Q}^{2}\right)\right]\left(k+m_{Q}\right)+\cdots\right\} .
\end{aligned}
$$

In the expressions above

$\begin{aligned} G_{a b}^{\alpha \beta} & =G_{A}^{\alpha \beta} t_{a b}^{A}, \quad G^{2}=G_{\alpha \beta}^{A} G_{\alpha \beta}^{A}, \\ G^{3} & =f^{A B C} G_{\mu \nu}^{A} G_{\nu \delta}^{B} G_{\delta \mu}^{C},\end{aligned}$

where $a, b=1,2,3$ are color indices and $A, B, C=$ $1,2, \ldots, 8$. Here $t^{A}=\lambda^{A} / 2$, where $\lambda^{A}$ are the Gell-Mann matrices, and the gluon field strength tensor is fixed at $x=0$, i.e. $G_{\alpha \beta}^{A} \equiv G_{\alpha \beta}^{A}(0)$.

The QCD sum rules can be derived after fixing the Lorentz structures in both the physical and the theoretical expressions of the correlation function and equating the correspondent invariant functions. In the case of the axial-vector particles the Lorentz structures in these expressions behave $\sim g_{\mu \nu}$ and $\sim p_{\mu} p_{\nu}$. Because the structures $\sim p_{\mu} p_{\nu}$ are contaminated 
by the scalar states with the same quark contents, we choose $\sim g_{\mu \nu}$ and the invariant function $\Pi^{\mathrm{QCD}}\left(p^{2}\right)$ corresponding to this structure. Then on the theoretical side of the sum rule there is only one invariant function $\Pi^{\mathrm{QCD}}\left(p^{2}\right)$, which can be represented as the dispersion integral

$$
\Pi^{\mathrm{QCD}}\left(p^{2}\right)=\int_{\mathcal{M}^{2}}^{\infty} \frac{\rho^{\mathrm{QCD}}(s)}{s-p^{2}} \mathrm{~d} s+\cdots,
$$

where the lower limit of the integral $\mathcal{M}$ in the case under consideration is equal to $\mathcal{M}=m_{b}+m_{c}$. When considering the $Z_{s}$ state it should be replaced by $\mathcal{M}=m_{b}+m_{c}+2 m_{s}$.

In Eq. (10), $\rho^{\mathrm{QCD}}(s)$ is the spectral density calculated as the imaginary part of the correlation function. It is the important component of the sum rule calculations. Because the technical tools necessary for derivation of $\rho^{\mathrm{QCD}}(s)$ in the case of the tetraquark states are well known and clearly explained in Refs. [47,52], here we avoid providing details of relevant manipulations, and refrain also from presenting explicit expressions for $\rho^{\mathrm{QCD}}(s)$. We want to emphasize only that the spectral density is computed by taking into account vacuum condensates up to dimension eight, and we include the effects of the quark $\langle\bar{q} q\rangle$, gluon $\left\langle\alpha_{s} G^{2} / \pi\right\rangle,\left\langle g_{s}^{3} G^{3}\right\rangle$, mixed $\left\langle\bar{q} g_{s} \sigma G q\right\rangle$ condensates, and also terms of their products.

Applying the Borel transformation on the variable $p^{2}$ to the invariant function $\Pi^{\mathrm{QCD}}\left(p^{2}\right)$, equating the obtained expression with $\mathcal{B}_{p^{2}} \Pi^{\text {Phys }}(p)$, and subtracting the contribution of higher resonances and continuum states, one finds the required sum rule. Then the sum rule for the mass of the $Z_{q}$ state reads

$m_{Z}^{2}=\frac{\int_{\mathcal{M}^{2}}^{s_{0}} \mathrm{~d} s \rho^{\mathrm{QCD}}(s) s e^{-s / M^{2}}}{\int_{\mathcal{M}^{2}}^{s_{0}} \mathrm{~d} s \rho^{\mathrm{QCD}}(s) e^{-s / M^{2}}}$.

The meson-current coupling $f_{Z}$ can be extracted from the sum rule:

$$
f_{Z}^{2} m_{Z}^{2} e^{-m_{Z}^{2} / M^{2}}=\int_{\mathcal{M}^{2}}^{s_{0}} \mathrm{~d} s \rho^{\mathrm{QCD}}(s) e^{-s / M^{2}} .
$$

In Eqs. (11) and (12) by $s_{0}$ we denote the threshold parameter, that separates the ground state's contribution from contributions arising due to higher resonances and continuum.

The sum rules contain the parameters which are necessary for numerical computations: Their numerical values are collected in Table 1. The quark and gluon condensates are well known, therefore we utilize their standard values. Table 1 contains also $B_{c}, \rho$ and $\phi$ mesons' masses (see Ref. [53]) and decay constants, which will serve as input parameters when computing the strong couplings and decay widths. It is worth noting that for $f_{\rho}, \phi$ and $f_{B_{c}}$ we use the sum rule estimations from Refs. [54,55].

The sum rules of Eqs. (11) and (12) contain also two parameters $s_{0}$ and $M^{2}$, choices of which are decisive to
Table 1 Input parameters

\begin{tabular}{ll}
\hline Parameters & Values \\
\hline$m_{B_{c}}$ & $(6275.1 \pm 1.0) \mathrm{MeV}$ \\
$f_{B_{c}}$ & $(528 \pm 19) \mathrm{MeV}$ \\
$m_{\rho}$ & $(775.26 \pm 0.25) \mathrm{MeV}$ \\
$f_{\rho}$ & $216 \pm 3 \mathrm{MeV}$ \\
$m_{\phi}$ & $(1019.461 \pm 0.019) \mathrm{MeV}$ \\
$f_{\phi}$ & $215 \pm 5 \mathrm{MeV}$ \\
$m_{b}$ & $4.18_{-0.03}^{+0.04} \mathrm{GeV}$ \\
$m_{c}$ & $(1.27 \pm 0.03) \mathrm{GeV}^{-}$ \\
$m_{s}$ & $96_{-4}^{+8} \mathrm{MeV}$ \\
$\langle\bar{q} q\rangle$ & $(-0.24 \pm 0.01)^{3} \mathrm{GeV}^{3}$ \\
$\langle\bar{s} s\rangle$ & $0.8\langle\bar{q} q\rangle$ \\
$m_{0}^{2}$ & $(0.8 \pm 0.1) \mathrm{GeV}^{2}$ \\
$\left\langle\bar{q} g_{s} \sigma G q\right\rangle$ & $m_{0}^{2}\langle\bar{q} q\rangle$ \\
$\left\langle\bar{s} g_{s} \sigma G s\right\rangle$ & $m_{0}^{2}\langle\bar{s} s\rangle$ \\
$\left\langle\frac{\alpha_{s} G^{2}}{\pi}\right\rangle$ & $(0.012 \pm 0.004) \mathrm{GeV}^{4}$ \\
$\left\langle g_{s}^{3} G^{3}\right\rangle$ & $(0.57 \pm 0.29) \mathrm{GeV}^{6}$ \\
\hline
\end{tabular}

extract reliable estimations for the quantities under question. The continuum threshold $s_{0}$ determines a boundary that dissects the ground-state contribution from ones due to excited resonances and continuum. It depends on the energy of the first excited state corresponding to the ground-state hadron. The continuum threshold $s_{0}$ can also be found from an analysis of the pole to total contribution ratio. The analysis done in the case of the tetraquark $Z_{q}$ allows us to fix a working interval for $s_{0}$ as

$59 \mathrm{GeV}^{2} \leq s_{0} \leq 60 \mathrm{GeV}^{2}$.

The Borel parameter $M^{2}$ has also to satisfy well-known requirements. Namely, convergence of OPE and exceeding of the perturbative contribution over the nonperturbative one fixes a lower bound of the allowed values of $M^{2}$. The upper limit of the Borel parameter is determined to achieve the largest possible pole contribution to the sum rule. These constraints lead to the following working window for $M^{2}$ :

$8.2 \mathrm{GeV}^{2} \leq M^{2} \leq 8.4 \mathrm{GeV}^{2}$.

In Figs. 1 and 2 we graphically demonstrate some stages in extracting of the working regions for these parameters. Thus, in Fig. 1 the perturbative and nonperturbative contributions to the sum rule in the chosen regions for $s_{0}$ and $M^{2}$ are depicted. The convergence of the OPE can be seen by inspecting Fig. 2, where the effects of the operators of the different dimensions are plotted. By varying the parameters $s_{0}$ and $M^{2}$ within their working ranges we find that the pole contribution to the mass sum rule amounts to $\sim 65 \%$ of the result. 

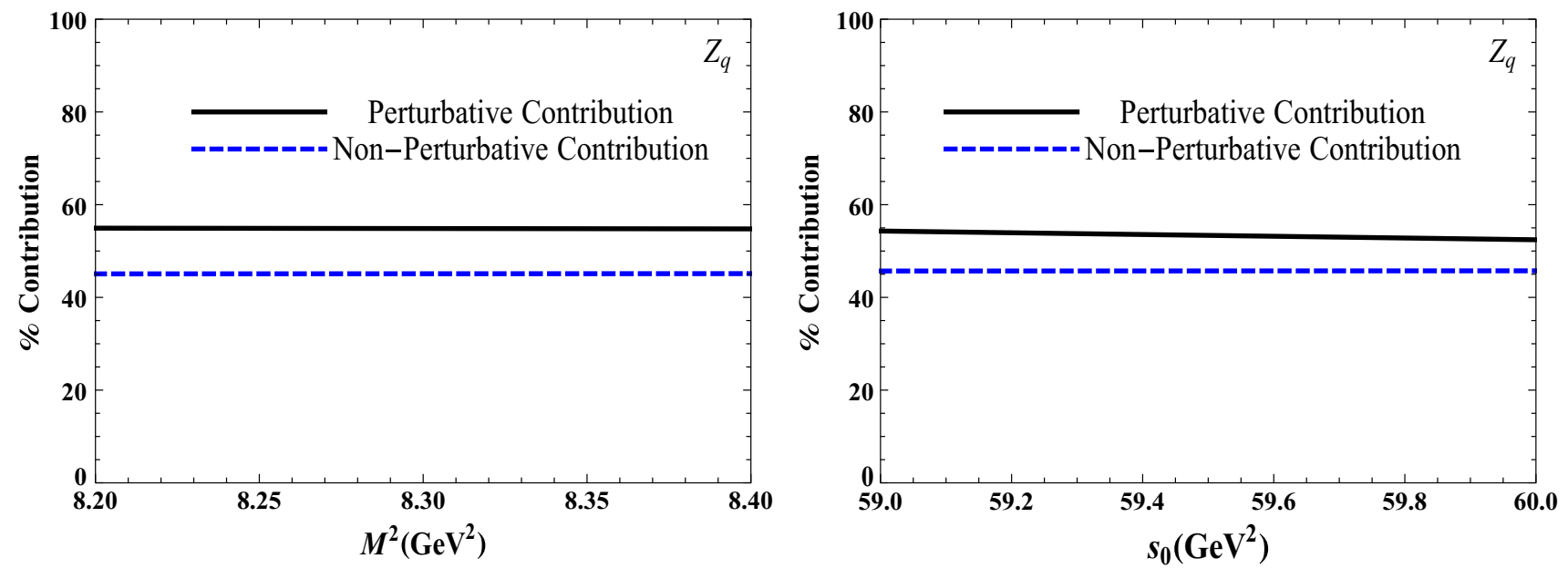

Fig. 1 The perturbative and nonperturbative contributions to the sum rule as functions of $M^{2}$ at an average $s_{0}$ (left panel), and as functions of $s_{0}$ at an average $M^{2}$ (right panel)
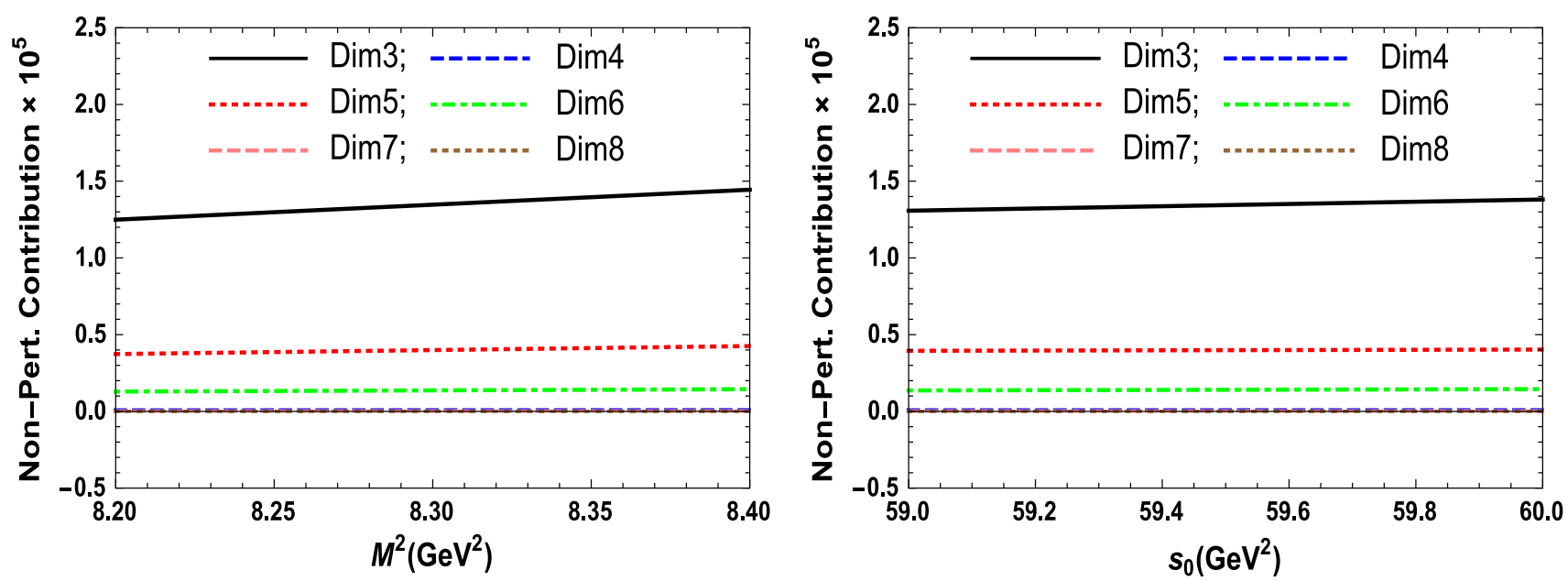

Fig. 2 Contributions to the sum rule arising from the nonperturbative operators of different dimensions are shown as functions of the Borel parameter at an average value of $s_{0}$ (left panel), and as functions of the threshold parameter $s_{0}$ at an average $M^{2}$ (right panel)

The final results for the mass and meson-current coupling of the $Z_{q}$ state are drawn in Fig. 3 and collected in Table 2. As is seen from Fig. 3 , the quantities extracted from the sum rules demonstrate a mild dependence on $M^{2}$, whereas the effects of $s_{0}$ on them are sizable. The uncertainties generated by the parameters $s_{0}$ and $M^{2}$ are main sources of errors, which are an inherent part of sum rule computations and equal to up to $30 \%$ of the whole integral.

The mass and meson-current coupling of the $Z_{s}$ state can be obtained from similar calculations, the difference being only in terms $\sim m_{s}$ kept in the spectral density, whereas in $Z_{q}$ calculations we set $m_{q}=0$. These modifications and also the replacement $\mathcal{M} \Rightarrow m_{b}+m_{c}+2 m_{s}$ in the integrals result in shifting of the working ranges of the parameters $s_{0}$ and $M^{2}$ towards slightly larger values, which now read

$$
60 \mathrm{GeV}^{2} \leq s_{0} \leq 61 \mathrm{GeV}^{2}
$$$$
8.4 \mathrm{GeV}^{2} \leq M^{2} \leq 8.6 \mathrm{GeV}^{2} \text {. }
$$

Predictions for $m_{Z_{s}}$ and $f_{Z_{s}}$ obtained using $s_{0}$ and $M^{2}$ from Eq. (15) are also written down in Table 2.

\section{$3 Z_{q} \rightarrow B_{c} \rho$ and $Z_{s} \rightarrow B_{c} \phi$ decays}

In this section we investigate the strong decays of the exotic axial-vector $Z_{q(s)}$ states, and calculate widths of their main decay modes, which, in accordance with results of Sect. 2, are kinematically allowed.

One can see that the quantum numbers, quark content and mass of the $Z_{q}$ tetraquark make the process $Z_{q} \rightarrow B_{c} \rho$ its preferable decay mode. The $Z_{s}$ state may decay to $B_{c}$ 

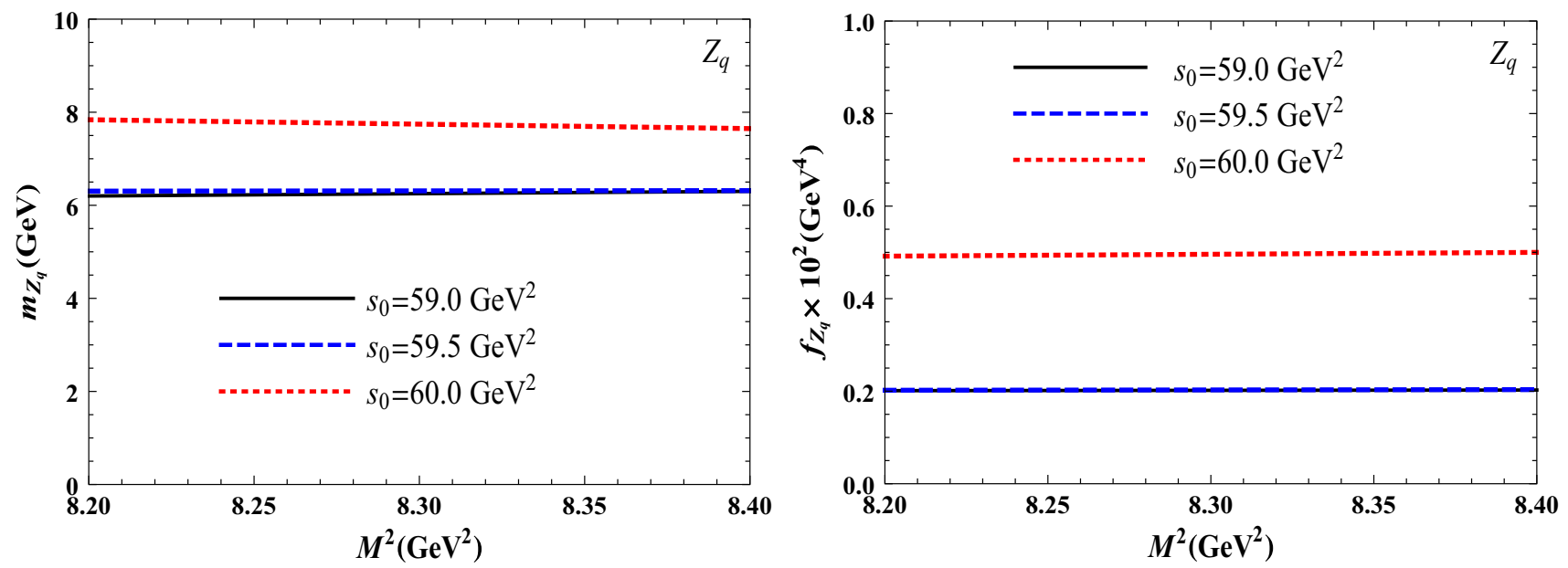

Fig. 3 The mass (left panel) and meson-current coupling (right panel) as functions of the Borel parameter $M^{2}$ at fixed values of the continuum threshold $s_{0}$

Table 2 The sum rule results for the masses and meson-current couplings of the axial-vector $Z_{q}$ and $Z_{s}$ states

\begin{tabular}{ll}
\hline Mass, m.-c. coupling & Results \\
\hline$m_{Z_{q}}$ & $(7.06 \pm 0.74) \mathrm{GeV}$ \\
$f_{Z_{q}}$ & $(0.33 \pm 0.11) \times 10^{-2} \mathrm{GeV}^{4}$ \\
$m_{Z_{s}}$ & $(7.30 \pm 0.76) \mathrm{GeV}$ \\
$f_{Z_{s}}$ & $(0.63 \pm 0.19) \times 10^{-2} \mathrm{GeV}^{4}$ \\
\hline
\end{tabular}

and $\phi$ mesons. It is worth noting that, due to $\rho-\omega$ and $\omega-$ $\phi$ mixing, the processes $Z_{q} \rightarrow B_{c} \omega$ and $Z_{s} \rightarrow B_{c} \omega$ are also among the kinematically allowed decay channels. But because, for example, the $\phi$ and $\omega$ mesons are almost pure $\bar{s} s$ and $(\bar{u} u+\bar{d} d) / \sqrt{2}$ states, the $Z_{s} \rightarrow B_{c} \omega$ process is unessential provided the mass of $Z_{s}$ allows its decay to $\phi$ meson. Alternative channels with $\omega$ may play an important role in the exploration of the tetraquark states containing an $\bar{s} s$ pair, if their masses are not enough to create a $\phi$ meson.

We are going to carry out the required analysis and write down all expressions necessary to find the $Z_{q} \rightarrow B_{c} \rho$ decay's width. After rather trivial replacements in the corresponding formulas and input parameters, the same calculations can easily be repeated for the $Z_{s} \rightarrow B_{c} \phi$ decay.

As a first step we have to compute the coupling $g_{Z_{q} B_{c} \rho}$, which describes the strong interaction in the vertex $Z_{q} B_{c} \rho$, and can be extracted from the QCD sum rule. To this end, we explore the correlation function

$\Pi_{\mu}(p, q)=i \int \mathrm{d}^{4} x e^{i p x}\left\langle\rho(q)\left|\mathcal{T}\left\{J^{B_{c}}(x) J_{\mu}^{\dagger}(0)\right\}\right| 0\right\rangle$,

where $J^{B_{c}}(x)$ is the interpolating current of the $B_{c}$ meson: It is defined in the form

$J^{B_{c}}(x)=i \bar{b}_{l}(x) \gamma_{5} c_{l}(x)$
The correlation function in Eq. (16) is introduced in a form which implies usage of the light-cone sum rule method. Indeed, $\Pi_{\mu}(p, q)$ will be computed employing the QCD sum rule on the light-cone by using the technique of the soft-meson approximation.

In terms of the physical parameters of the involved particles and coupling $g_{Z_{q} B_{c} \rho}$, the function $\Pi_{\mu}(p, q)$ has a simple form and generates the phenomenological side of the sum rule. Namely,

$$
\begin{aligned}
\Pi_{\mu}^{\text {Phys }}(p, q)= & \frac{\left\langle 0\left|J^{B_{c}}\right| B_{c}(p)\right\rangle}{p^{2}-m_{B_{c}}^{2}}\left\langle B_{c}(p) \rho(q) \mid Z_{q}\left(p^{\prime}\right)\right\rangle \\
& \times \frac{\left\langle Z_{q}\left(p^{\prime}\right)\left|J_{\mu}^{\dagger}\right| 0\right\rangle}{p^{\prime 2}-m_{Z}^{2}}+\cdots,
\end{aligned}
$$

where $p, q$ and $p^{\prime}=p+q$ are the momenta of $B_{c}, \rho$ and $Z_{q}$ particles, respectively. The term presented above is the contribution of the ground state: the dots stand for the effects of the higher resonances and continuum states.

We introduce the $B_{c}$ meson matrix element,

$\left\langle 0\left|J^{B_{c}}\right| B_{c}(p)\right\rangle=\frac{f_{B_{c}} m_{B_{c}}^{2}}{m_{b}+m_{c}}$

where $m_{B_{c}}$ and $f_{B_{c}}$ are the mass and decay constant of the $B_{c}$ meson, and also the matrix element corresponding to the vertex

$$
\begin{aligned}
\left\langle B_{c}(p) \rho(q) \mid Z_{q}\left(p^{\prime}\right)\right\rangle= & g_{Z_{q} B_{c} \rho}\left[\left(q \cdot \varepsilon^{\prime}\right)\left(p^{\prime} \cdot \varepsilon^{*}\right)\right. \\
& \left.-\left(q \cdot p^{\prime}\right)\left(\varepsilon^{*} \cdot \varepsilon^{\prime}\right)\right] .
\end{aligned}
$$

Then the ground-state term in the correlation function can easily be found: 


$$
\begin{aligned}
\Pi_{\mu}^{\text {Phys }}(p, q)= & \frac{f_{B_{c}} f_{Z} m_{Z} m_{B_{c}}^{2} g_{Z_{q} B_{c} \rho}}{\left(p^{\prime 2}-m_{Z}^{2}\right)\left(p^{2}-m_{B_{c}}^{2}\right)\left(m_{b}+m_{c}\right)} \\
& \times\left(\frac{m_{Z}^{2}-m_{B_{c}}^{2}}{2} \varepsilon_{\mu}^{*}-p^{\prime} \cdot \varepsilon^{*} q_{\mu}\right)+\cdots .
\end{aligned}
$$

Strong vertices of a tetraquark with two conventional mesons differ from vertices containing only ordinary mesons. The reason here is very simple: the tetraquark $Z_{q}$ is a state composed of four valence quarks, therefore the expansion of the non-local correlation function $\Pi_{\mu}(p, q)$ leads to the expression, which instead of distribution amplitudes of $\rho$ meson depends on its local matrix elements (of course, same arguments are valid for $Z_{s}$, as well). Then the conservation of the four-momentum at the vertex $Z_{q} B_{c} \rho$ equals $q$ to zero. In other words, within the light-cone sum rule method the momentum of $\rho$ meson should be equal to zero in our case. In the vertices of ordinary hadrons the four-momenta of all involved particles can take nonzero values. The soft-meson approximation corresponds to a situation when $q=0$. Calculations of the same strong couplings within the full lightcone sum rule method and in the soft-meson approximation demonstrated that the difference between the results extracted using these two approaches is numerically small (for a detailed discussion, see Ref. [46]).

In the soft limit $p=p^{\prime}$, the only term that survives in Eq. (20) is $\sim \varepsilon_{\mu}^{*}$. The invariant function $\Pi^{\text {Phys }}\left(p^{2}\right)$ corresponding to this structure depends on the variable $p^{2}$ and is given as

$$
\begin{aligned}
\Pi^{\text {Phys }}\left(p^{2}\right)= & \frac{f_{B_{c}} f_{Z} m_{Z} m_{B_{c}}^{2} g_{Z_{s} B_{c} \eta}}{2\left(p^{2}-m^{2}\right)^{2}\left(m_{b}+m_{c}\right)} \\
& \times\left(m_{Z}^{2}-m_{B_{c}}^{2}\right)+\cdots,
\end{aligned}
$$

where $m^{2}=\left(m_{Z}^{2}+m_{B_{c}}^{2}\right) / 2$.

In the soft-meson approximation we additionally apply the operator

$$
\left(1-M^{2} \frac{\mathrm{d}}{\mathrm{d} M^{2}}\right) M^{2} e^{m^{2} / M^{2}}
$$

to both sides of the sum rule. The last operation is required to remove all unsuppressed contributions existing on the physical side of the sum rule in the soft-meson limit (see Ref. [45]).

The second component of the sum rule, i.e. the QCD expression for the correlation function $\Pi_{\mu}^{\mathrm{QCD}}(p, q)$, is calculated employing the quark propagators:

$$
\begin{aligned}
& \Pi_{\mu}^{\mathrm{QCD}}(p, q)=-i \int \mathrm{d}^{4} x e^{i p x}\left\{\left[\gamma_{5} \widetilde{S}_{c}^{i b}(x) \gamma_{5}\right.\right. \\
& \left.\quad \times \widetilde{S}_{b}^{b i}(-x) \gamma_{\mu}\right]_{\alpha \beta}\left\langle\rho(q)\left|\bar{q}_{\alpha}^{a} q_{\beta}^{a}\right| 0\right\rangle \\
& \left.\quad+\left[\gamma_{5} \widetilde{S}_{c}^{i b}(x) \gamma_{5} \widetilde{S}_{b}^{a i}(-x) \gamma_{\mu}\right]_{\alpha \beta}\left\langle\rho(q)\left|\bar{s}_{\alpha}^{a} s_{\beta}^{b}\right| 0\right\rangle\right\},
\end{aligned}
$$

with $\alpha$ and $\beta$ being the spinor indices.

We continue our calculations by employing the expansion

$\bar{q}_{\alpha}^{a} q_{\beta}^{b} \rightarrow \frac{1}{4} \Gamma_{\beta \alpha}^{j}\left(\bar{q}^{a} \Gamma^{j} q^{b}\right)$

where $\Gamma^{j}=1, \gamma_{5}, \gamma_{\mu}, i \gamma_{5} \gamma_{\mu}, \sigma_{\mu \nu} / \sqrt{2}$ is the full set of Dirac matrices, and we carry out the color summation.

Prescriptions to perform summation over color indices, as well as procedures to calculate the resulting integrals and extract the imaginary part of the correlation function $\Pi_{\mu}^{\mathrm{QCD}}(p, q)$, were numerously presented in our previous work; see Refs. [47-50]. Therefore, here we skip further details, and we provide the $\rho$ meson local matrix elements that in the soft limit contribute to the spectral density, as well as the final formulas for the spectral density $\rho_{c}(s)$.

Analysis demonstrates that in the soft limit only the matrix elements

$\left\langle 0\left|\bar{q} \gamma_{\mu} q\right| \rho^{0}(p)\right\rangle=\frac{1}{\sqrt{2}} f_{\rho} m_{\rho} \varepsilon_{\mu}$

and

$\left\langle 0\left|\bar{q} g \widetilde{G}_{\mu \nu} \gamma_{\nu} \gamma_{5} q\right| \rho^{0}(p)\right\rangle=\frac{1}{\sqrt{2}} f_{\rho} m_{\rho}^{3} \zeta_{4 \rho} \varepsilon_{\mu}$

are involved in the computations, where $q$ denotes one of the $u$ or $d$ quarks. The matrix elements depend on the $\rho$ meson mass $m_{\rho}$ and decay constant $f_{\rho}$. The twist-4 matrix element in Eq. (26), as a factor, contains also the parameter $\zeta_{4 \rho}$. Its numerical value was extracted at the scale $\mu=1 \mathrm{GeV}$ from the sum rule calculations in Ref. [54] and equals

$\zeta_{4 \rho}=0.07 \pm 0.03$

The final expression of the spectral density has the form

$\rho_{c}(s)=\frac{f_{\rho} m_{\rho}}{24 \sqrt{2}}\left[F^{\text {pert. }}(s)+F^{\text {n.-pert. }}(s)\right]$.

Here $F^{\text {pert. }}(s)$ is the perturbative contribution to $\rho_{c}(s)$,

$$
\begin{aligned}
F^{\text {pert. }}(s)= & \frac{1}{\pi^{2} s^{2}}\left\{\left[s^{2}+s\left(m_{b}^{2}+6 m_{b} m_{c}+m_{c}^{2}\right)\right.\right. \\
& \left.\left.-2\left(m_{b}^{2}-m_{c}^{2}\right)^{2}\right]\right\} \sqrt{\left(s+m_{b}^{2}-m_{c}^{2}\right)^{2}-4 m_{b}^{2} s}
\end{aligned}
$$


whereas by $F^{\text {n.-pert. }}(s)$ we denote its nonperturbative component. The function $F^{\text {n.-pert. }}(s)$ is the sum of the terms

$$
\begin{aligned}
F^{\text {n. }- \text { pert. }}(s)= & F_{G}^{\text {n. }- \text { pert. }}(s)+\left\langle\frac{\alpha_{s} G^{2}}{\pi}\right\rangle \int_{0}^{1} f_{g_{s}^{2} G^{2}}(z, s) \mathrm{d} z \\
& +\left\langle g_{s}^{3} G^{3}\right\rangle \int_{0}^{1} f_{g_{s}^{3} G^{3}}(z, s) \mathrm{d} z \\
& +\left\langle\frac{\alpha_{s} G^{2}}{\pi}\right\rangle^{2} \int_{0}^{1} f_{\left(g_{s}^{2} G^{2}\right)^{2}}(z, s) \mathrm{d} z .
\end{aligned}
$$

Here $F_{G}^{\text {n.-pert. }}(s)$ appears from the integration of the perturbative component of one heavy quark propagator with the term $\sim G$ from another one. It can be expressed using the matrix element given by Eq. (26) and has a rather simple form:

$F_{G}^{\text {n. }- \text { pert. }}(s)=\frac{3 m_{\rho}^{2} \zeta_{4 \rho}}{2 \pi^{2} s} \sqrt{\left(s+m_{b}^{2}-m_{c}^{2}\right)^{2}-4 m_{b}^{2} s}$.

The nonperturbative factors in front of the integrals and the subscripts of the functions clearly indicate the origin of the remaining terms. In fact, the functions $f_{g_{s}^{2} G^{2}}, f_{g_{s}^{3} G^{3}}$ are due to products of terms $\sim g_{s}^{2} G^{2}$ and $\sim g_{s}^{3} G^{3}$ with the perturbative component of another propagator, whereas $f_{\left(g_{s}^{2} G^{2}\right)^{2}}$ comes from integrals obtained using $\sim g_{s}^{2} G^{2}$ components of $b$ and $c$ quarks' propagators. These terms are four, six and eight dimensional nonperturbative contributions to the spectral density $\rho_{c}(s)$, respectively. Their explicit forms are

$$
\begin{aligned}
& f_{g_{s}^{2} G^{2}}(z, s)=\frac{1}{12 z^{2}(z-1)^{2}}\left\{54(1-z) z^{2} \delta(s-\Phi)\right. \\
& \quad+\left[8 m_{b}^{2}(z-1)^{3}+z^{2}\left(27 s(1-z)-8 m_{b}^{2} z\right)\right. \\
& \left.\quad+2 m_{b} m_{c}\left(4+15 z+12 z^{2}\right)\right] \delta^{(1)}(s-\Phi) \\
& \left.\quad-4 s\left[m_{b}^{2}(1-z)^{3}+m_{b} m_{c} z(1-z)-m_{c}^{2} z^{3}\right] \delta^{(2)}(s-\Phi)\right\},
\end{aligned}
$$

$$
\begin{aligned}
& f_{g_{s}^{3} G^{3}}(z, s)=\frac{1}{15 \cdot 2^{6} z^{5}(z-1)^{5}}\left\{-12 z^{2}(z-1)^{2}\left[3 m_{b}^{2}(z-1)^{5}\right.\right. \\
& \quad+3 m_{b} m_{c}\left((1-z)^{5}+z^{5}\right)+z\left(-3 m_{c}^{2} z^{4}\right. \\
& \left.\left.\quad+s\left(1-8 z+25 z^{2}-40 z^{3}+33 z^{4}-11 z^{5}\right)\right)\right] \delta^{(2)}(s-\Phi) \\
& \quad+2 z(z-1)\left[m_{b}^{2}(z-1)^{5}\left(7 m_{b}^{2}-4 m_{b} m_{c}-9 s z(2 z-1)\right)\right. \\
& \quad+2 m_{b} m_{c} z^{2}\left(2 m_{c}^{2} z^{3}-9 s(z-1)^{2}\left(1-3 z+3 z^{2}\right)\right) \\
& +z^{3}\left(-7 m_{c}^{4} z^{2}+9 s m_{c}^{2} z^{2}\left(1-3 z+2 z^{2}\right)\right. \\
& \left.\left.\quad+2 s^{2}(z-1)^{3}\left(2-7 z+7 z^{2}\right)\right)\right] \\
& \quad \times \delta^{(3)}(s-\Phi)+\left[-2 m_{b}^{5} m_{c}(z-1)^{5}+7 m_{b}^{4} s z(z-1)^{6}\right. \\
& -4 m_{b}^{3} m_{c} s z^{2}(z-1)^{5}-6 m_{b}^{2} s^{2} z^{3}(z-1)^{6}+2 m_{b} m_{c} z^{4}
\end{aligned}
$$

$$
\begin{aligned}
& \times\left(-3 s^{2}(z-1)^{4}+m_{c}^{4} z+2 m_{c}^{2} s z(z-1)^{2}\right)+s(z-1) z^{5} \\
& \left.\left.\times\left(s^{2}(z-1)^{4}-7 m_{c}^{4} z+6 m_{c}^{2} s z(z-1)^{2}\right)\right] \delta^{(4)}(s-\Phi)\right\}, \\
& f_{\left(g_{s}^{2} G^{2}\right)^{2}}(z, s)=\frac{m_{b} m_{c}}{54 z^{2}(z-1)^{2}}\left\{2\left[m_{b} m_{c}-s\left(1-3 z+3 z^{2}\right)\right]\right. \\
& \left.\times \delta^{(4)}(s-\Phi)+s\left[m_{b} m_{c}+s(1-z) z\right] \delta^{(5)}(s-\Phi)\right\},
\end{aligned}
$$

where

$\delta^{(n)}(s-\Phi)=\frac{\mathrm{d}^{n}}{\mathrm{~d} s^{n}} \delta(s-\Phi)$,

with $\Phi$ being defined as

$\Phi=\frac{m_{b}^{2}(1-z)+m_{c}^{2} z}{z(1-z)}$.

The final sum rule to evaluate the strong coupling reads

$$
\begin{aligned}
g_{Z_{q} B_{c} \rho}= & \frac{2\left(m_{b}+m_{c}\right)}{f_{B_{c}} f_{Z} m_{Z} m_{B_{c}}^{2}\left(m_{Z}^{2}-m_{B_{c}}^{2}\right)}\left(1-M^{2} \frac{\mathrm{d}}{\mathrm{d} M^{2}}\right) \\
& \times M^{2} \int_{\left(m_{b}+m_{c}\right)^{2}}^{s_{0}} \mathrm{~d} s e^{\left(m^{2}-s\right) / M^{2}} \rho_{c}(s) .
\end{aligned}
$$

To calculate the width of the decay $Z_{q} \rightarrow B_{c} \rho$ we use the expression

$$
\begin{aligned}
\Gamma\left(Z_{q} \rightarrow B_{c} \rho\right)= & \frac{g_{Z_{q} B_{c} \rho}^{2} m_{\rho}^{2}}{24 \pi} \lambda\left(m_{Z}, m_{B_{c}}, m_{\rho}\right) \\
& \times\left[3+\frac{2 \lambda^{2}\left(m_{Z_{q}}, m_{B_{c}}, m_{\rho}\right)}{m_{\rho}^{2}}\right],
\end{aligned}
$$

where

$\lambda(a, b, c)=\frac{\sqrt{a^{4}+b^{4}+c^{4}-2\left(a^{2} b^{2}+a^{2} c^{2}+b^{2} c^{2}\right)}}{2 a}$.

The parameters necessary for numerical calculations of the strong coupling $g_{Z_{q} B_{c} \rho}$ and $\Gamma\left(Z_{q} \rightarrow B_{c} \rho\right)$ are listed in Table 1.

The investigation carried out in accordance with standard requirements of the sum rule calculations allows us to determine the ranges for $s_{0}$ and $M^{2}$. For example, the pole contribution to the sum rule amounts to $\sim 48$ to $60 \%$ of the total result, as is seen from Fig. 4. Other constraints, i.e. convergence of the OPE and prevalence of the perturbative contribution, have been checked, as well. Summing up the performed analysis we fix the interval for the continuum threshold $s_{0}$ as in the mass calculations [see Eq. (13)], whereas for the Borel parameter we obtain

$8 \mathrm{GeV}^{2} \leq M^{2} \leq 9 \mathrm{GeV}^{2}$, 

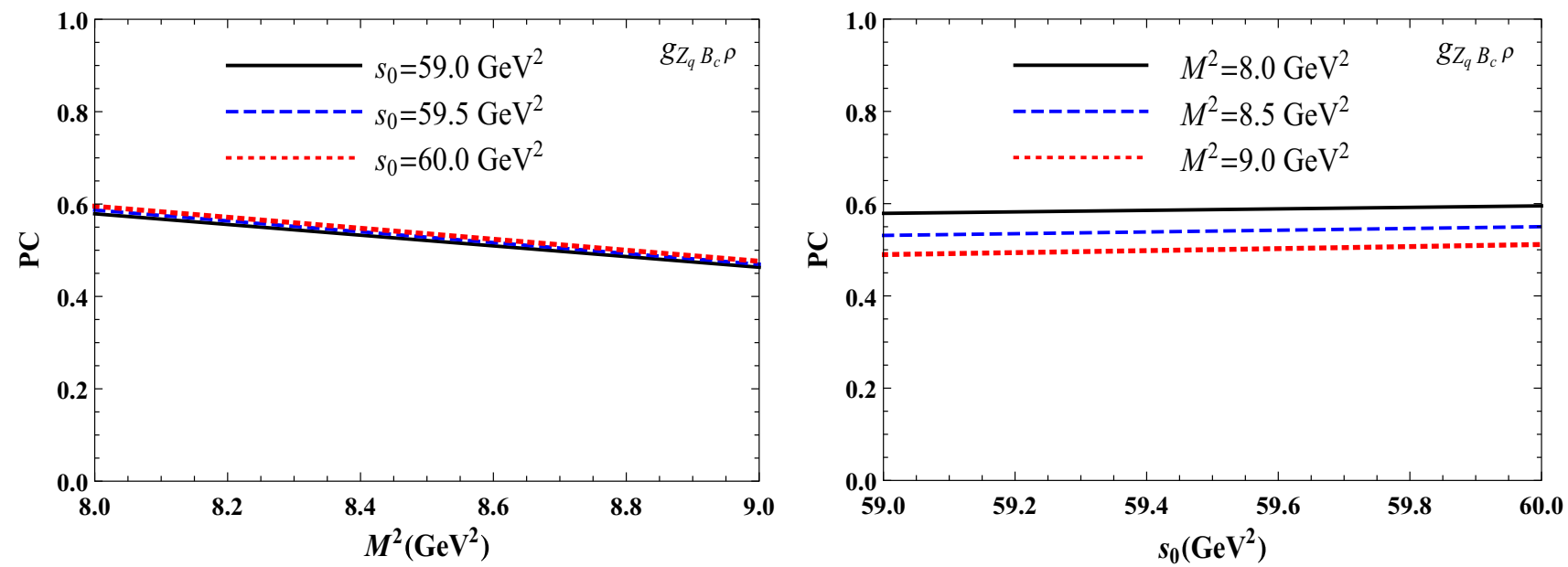

Fig. 4 The pole contribution in the $g_{Z_{q} B_{c} \rho}$ coupling sum rule calculations as a function of the Borel parameter $M^{2}$ at fixed $s_{0}$ (left panel), and as a function of the threshold $s_{0}$ at fixed values of $M^{2}$ (right panel)
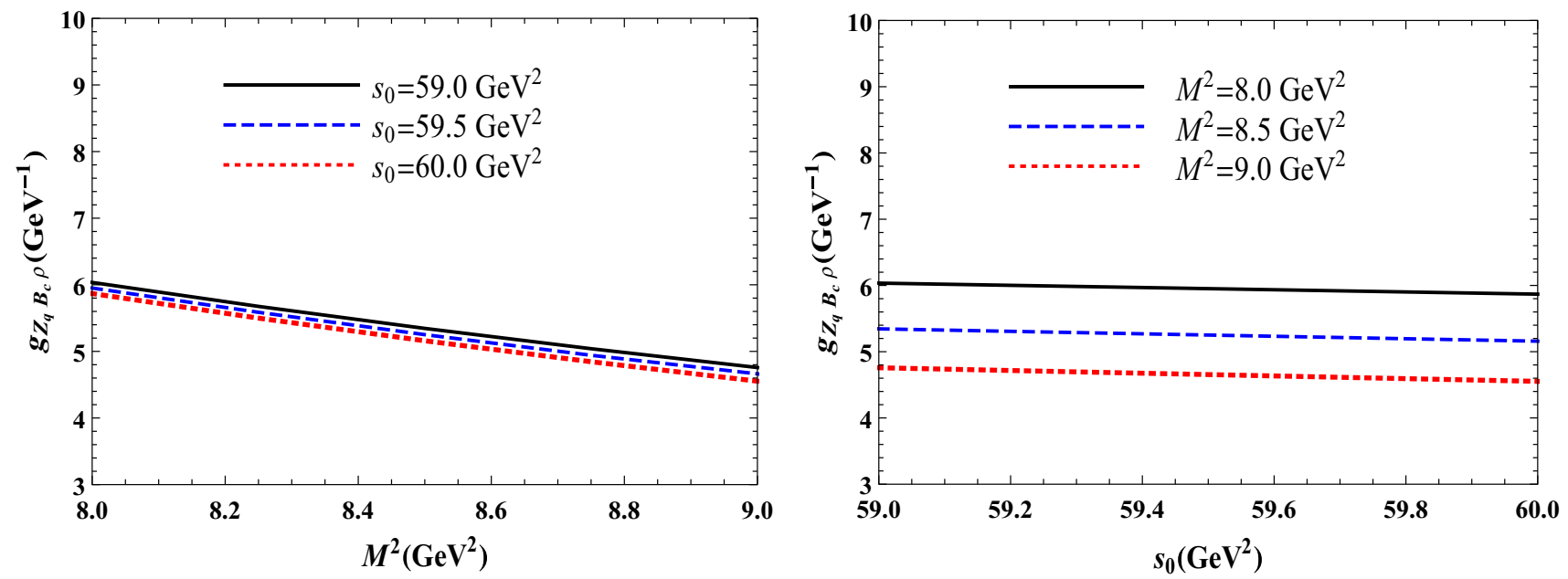

Fig. 5 The strong coupling $g_{Z_{q} B_{c} \rho}$ as a function of the Borel parameter $M^{2}$ at fixed $s_{0}$ (left panel), and as a function of the threshold $s_{0}$ at fixed values of $M^{2}$ (right panel)

which is wider than the corresponding window in the mass sum rule.

In Fig. 5 we provide our final results and depict the strong coupling $g_{Z_{q} B_{c} \rho}$ as a function of the Borel parameter (at fixed $s_{0}$ ) and as a function of the continuum threshold (at fixed $M^{2}$ ). The dependence of the strong coupling on these parameters has a traditional form, and systematic errors of the calculations are within reasonable limits.

The decay $Z_{s} \rightarrow B_{c} \phi$ can be considered in analogous manner: One only needs to write down in the relevant expressions the parameters of the $\phi$ meson. Thus, the matrix elements of the $\phi$ meson that take part in forming of the spectral density are$$
\left\langle 0\left|\bar{s} \gamma_{\mu} s\right| \phi(p)\right\rangle=f_{\phi} m_{\phi} \varepsilon_{\mu},
$$$$
\left\langle 0\left|\bar{s} g \widetilde{G}_{\mu \nu} \gamma_{\nu} \gamma_{5} s\right| \phi(p)\right\rangle=f_{\phi} m_{\phi}^{3} \zeta_{4 \phi} \varepsilon_{\mu},
$$

where the twist- 4 parameter

$\zeta_{4 \phi}=0.00 \pm 0.02$

was estimated and found to be compatible with zero in Ref. [54].

In calculations of the coupling $g_{Z_{s} B_{c} \phi}$ the working regions for the Borel parameter and continuum threshold are fixed in the form

$$
\begin{aligned}
60 \mathrm{GeV}^{2} & \leq s_{0} \leq 61 \mathrm{GeV}^{2} \\
8.2 \mathrm{GeV}^{2} & \leq M^{2} \leq 9.2 \mathrm{GeV}^{2}
\end{aligned}
$$

Our results for the strong couplings and widths of the decay modes studied in this work are collected in Table 3. 
Table 3 The strong couplings and decay widths of the $Z_{q}$ and $Z_{s}$ tetraquarks

\begin{tabular}{ll}
\hline Strong couplings, widths & Predictions \\
\hline$g_{Z_{q} B_{c} \rho}$ & $(5.31 \pm 1.25) \mathrm{GeV}^{-1}$ \\
$g_{Z_{s} B_{c} \phi}$ & $(6.42 \pm 1.52) \mathrm{GeV}^{-1}$ \\
$\Gamma\left(Z_{q} \rightarrow B_{c} \rho\right)$ & $(80 \pm 32) \mathrm{MeV}$ \\
$\Gamma\left(Z_{s} \rightarrow B_{c} \phi\right)$ & $(168 \pm 68) \mathrm{MeV}$ \\
\hline
\end{tabular}

\section{Discussion and concluding remarks}

In the present work we have calculated the parameters of the open charm-bottom axial-vector tetraquark states $Z_{q}$ and $Z_{s}$ within the QCD sum rule method. Their masses and mesoncurrent couplings have been obtained using the two-point sum rule method. In these calculations for $Z_{q}$ and $Z_{s}$ we have used the symmetric in color indices interpolating currents by assuming that they are ground states in the corresponding tetraquark multiplets. Indeed, one can anticipate that $Z_{q}$ and $Z_{s}$ are the axial-vector components of the $1 S$ diquarkantidiquark $[c q][\bar{b} \bar{q}]$ and $[c s][\bar{b} \bar{s}]$ multiplets, respectively.

During last years some progress was achieved in the investigation of the $[c q][\bar{c} \bar{q}]$ and $[c s][\bar{c} \bar{s}]$ multiplets, and classification of the observed hidden-charm tetraquarks as their possible members (see Refs. [56,57]). Thus, within the "type-II" model elaborated in this work, the authors not only identified the multiplet levels with the tetraquarks discovered, but also the estimated masses of the states, which had not yet been observed. This model is founded on some assumptions as regards the nature of inter-quark and inter-diquark interactions, and one considers spin-spin interactions within diquarks as the decisive source of splitting inside of the multiplet.

The information useful for our purposes is accumulated in the axial-vector sector of these multiplets. The axial-vector $J^{P C}=1^{++}$particle in the ground-state $[c q][\bar{c} \bar{q}]$ multiplet was identified with the well-known $X(3872)$ resonance. A similar analysis carried out for the multiplet of $[c s][\bar{c} \bar{s}]$ states demonstrated that its $J^{P C}=1^{++}$level may be considered as $X(4140)$. The mass difference of the axial-vector resonances belonging to " $q$ " and " $s$ " hidden-charm multiplets is

$X(4140)-X(3872) \approx 270 \mathrm{MeV}$.

In the present work we have evaluated the masses of the axialvector states from the $[c q][\bar{b} \bar{q}]$ and $[c s][\bar{b} \bar{s}]$ multiplets. The mass shift between these multiplets,

$m_{Z_{s}}-m_{Z_{q}} \approx 240 \mathrm{MeV}$,

is in nice agreement with Eq. (38).

Another question to be addressed here is connected with the masses of the excited states, which in sum rule cal- culations determine the continuum threshold $s_{0}$. We have found that, for the $[c q][\bar{b} \bar{q}]$ and $[c s][\bar{b} \bar{s}]$ multiplets, sum rule calculations fix the lower bounds of the parameter $s_{0}$ as $s_{0}=59 \mathrm{GeV}^{2}$ and $s_{0}=60 \mathrm{GeV}^{2}$, respectively. This means that the sum rule has placed a first excited state to the position $\sqrt{s_{0}}$. In order to estimate the gap between the excited and ground states we invoke $\sqrt{s_{0}}$ and the central values of $Z_{q}$ and $Z_{s}$ masses. Then it is not difficult to see that, for the $[c q][\bar{b} \bar{q}]$ type tetraquarks, it equals

$\sqrt{s_{0}} \mathrm{GeV}-7.06 \mathrm{GeV} \approx 0.62 \mathrm{GeV}$,

whereas for the $[c s][\bar{b} \bar{s}]$ one gets

$\sqrt{s_{0}} \mathrm{GeV}-7.30 \mathrm{GeV} \approx 0.45 \mathrm{GeV}$.

The masses of the $1 S$ and $2 S$ states with $J^{P C}=1^{+-}$from the $[c q]\left[\bar{c} \bar{q}^{\prime}\right]$ multiplet were calculated by means of the twopoint sum rule method in Ref. [58]. The ground-state level $1 S$ was identified with the resonance $Z_{c}$ (3900), whereas the resonance $Z$ (4430) was included into a multiplet of the excited $2 S$ states. If this assignment is correct, then the experimental data provides the mass difference between the ground and first radially excited states, which is equal to $530 \mathrm{MeV}$. The results of the calculations led to the predictions $M_{Z_{c}(3900)}=$ $3.91_{-17}^{+21} \mathrm{GeV}$ and $M_{Z_{c}(4430)}=4.51_{-09}^{+17} \mathrm{GeV}$, and to the mass difference $\sim 600 \mathrm{MeV}$.

The $1 S$ and $2 S$ multiplets of the $[c s][\bar{c} \bar{s}]$ tetraquarks were explored in the context of the "type-II" model in Ref. [57]. For the axial-vector levels $J^{P C}=1^{++}$, there called $X$ states, the $2 S-1 S$ gap is $4600 \mathrm{MeV}-4140 \mathrm{MeV}=460 \mathrm{MeV}$, and for the particles $X^{(1)}$ and $X^{(2)}$ with the quantum numbers $J^{P C}=1^{+-}$one gets $4600 \mathrm{MeV}-4140 \mathrm{MeV}=460 \mathrm{MeV}$ and $4700 \mathrm{MeV}-4274 \mathrm{MeV}=426 \mathrm{MeV}$, respectively. Comparison of these results with the ones given by Eqs. (40) and (41) can be considered as confirmation of the self-consistent character of the performed analysis.

In the framework of the QCD two-point sum rule approach, the masses of the open charm-bottom diquarkantidiquark states were previously calculated in Ref. [30]. For the masses of the axial-vector tetqaruarks $Z_{q}$ and $Z_{s}$ the authors found

$m_{Z_{q}}=7.10 \pm 0.09 \pm 0.06 \pm 0.01 \mathrm{GeV}$

and

$m_{Z_{s}}=7.11 \pm 0.08 \pm 0.05 \pm 0.03 \mathrm{GeV}$.

These predictions were extracted by using the parameter $s_{0}=(55 \pm 2) \mathrm{GeV}^{2}$ in calculations of $m_{Z_{q}}$ and $m_{Z_{s}}$, and $M^{2}=(7.9-8.2) \mathrm{GeV}^{2}$ and $M^{2}=(6.7-7.9) \mathrm{GeV}^{2}$ for the " $q$ " and " $s$ " states, respectively. It is seen that the 
mass differences $m_{Z_{s}}-m_{Z_{q}} \approx 10 \mathrm{MeV}$ and $\sqrt{s_{0}}-m_{Z_{q}} \approx$ $\sqrt{s_{0}}-m_{Z_{s}} \approx 180 \mathrm{MeV}$ can be neither included into the " $q$ "" $s$ " mass-hierarchy scheme of the ground-state tetraquarks, nor accepted as giving a correct mass shift between the $1 S$ and $2 S$ multiplets. Our results for $m_{Z_{q}}$ and $m_{Z_{s}}$, if differences are ignored in the chosen windows for the parameters $s_{0}$ and $M^{2}$, within theoretical errors may be considered as being in agreement with the predictions of Ref. [30]. But in our case the central value of $m_{Z_{s}}$ allows the decay process $Z_{s} \rightarrow B_{c} \phi$, whereas for $m_{Z_{s}}$ from Eq. (43) it remains among the kinematically forbidden channels.

We have also calculated the widths of the $Z_{q} \rightarrow B_{c} \rho$ and $Z_{s} \rightarrow B_{c} \phi$ decays, which are new results of this work. The obtained predictions for $\Gamma\left(Z_{q} \rightarrow B_{c} \rho\right)$ and $\Gamma\left(Z_{s} \rightarrow\right.$ $B_{c} \phi$ ) show that $Z_{q}$ may be considered as a narrow resonance, whereas $Z_{s}$ belongs to a class of wide tetraquark states.

Investigation of the open charm-bottom axial-vector tetraquarks performed in the present work within the diquarkantidiquark picture led to quite interesting predictions. Theoretical explorations of other members of the $[c q][\bar{b} \bar{q}]$ and $[c s][\bar{b} \bar{s}]$ tetraquark multiplets, as well as their experimental studies, may shed light on the nature of multi-quark hadrons.

Acknowledgements The work of K. A. was financed by TUBITAK under the Grant No. 115F183.

Open Access This article is distributed under the terms of the Creative Commons Attribution 4.0 International License (http://creativecomm ons.org/licenses/by/4.0/), which permits unrestricted use, distribution, and reproduction in any medium, provided you give appropriate credit to the original author(s) and the source, provide a link to the Creative Commons license, and indicate if changes were made.

Funded by SCOAP ${ }^{3}$.

\section{References}

1. H.X. Chen, W. Chen, X. Liu, S.L. Zhu, Phys. Rep. 639, 1 (2016)

2. H.X. Chen, W. Chen, X. Liu, Y.R. Liu, S.L. Zhu. arXiv:1609.08928 [hep-ph]

3. A. Esposito, A.L. Guerrieri, F. Piccinini, A. Pilloni, A.D. Polosa, Int. J. Mod. Phys. A 30, 1530002 (2015)

4. C.A. Meyer, E.S. Swanson, Prog. Part. Nucl. Phys. 82, 21 (2015)

5. S.-K. Choi et al. [Belle Collaboration], Phys. Rev. Lett. 91, 262001 (2003)

6. D. Acosta et al. [CDF II Collaboration], Phys. Rev. Lett. 93, 072001 (2004)

7. V.M. Abazov et al. [D0 Collaboration], Phys. Rev. Lett. 93, 162002 (2004)

8. B. Aubert et al. [BaBar Collaboration], Phys. Rev. D 71, 071103 (2005)

9. K. Abe et al. [Belle Collaboration], BELLE-CONF-0540. arXiv:hep-ex/0505037

10. B. Aubert et al. [BaBar Collaboration], Phys. Rev. Lett. 102, 132001 (2009)

11. T. Barnes, S. Godfrey, E.S. Swanson, Phys. Rev. D 72, 054026 (2005)

12. I.V. Danilkin, Y.A. Simonov, Phys. Rev. Lett. 105, 102002 (2010)

13. F.E. Close, P.R. Page, Phys. Lett. B 578, 119 (2004)
14. N.A. Tornqvist, Phys. Lett. B 590, 209 (2004)

15. C.M. Zanetti, M. Nielsen, R.D. Matheus, Phys. Lett. B 702, 359 (2011)

16. F.K. Guo, C. Hanhart, Y.S. Kalashnikova, U.G. Meißner, A.V. Nefediev, Phys. Lett. B 742, 394 (2015)

17. L. Maiani, F. Piccinini, A.D. Polosa, V. Riquer, Phys. Rev. D 71, 014028 (2005)

18. L. Maiani, A.D. Polosa, V. Riquer, Phys. Rev. Lett. 99, 182003 (2007)

19. F.S. Navarra, M. Nielsen, Phys. Lett. B 639, 272 (2006)

20. S. Dubnicka, A.Z. Dubnickova, M.A. Ivanov, J.G. Korner, Phys. Rev. D 81, 114007 (2010)

21. Z.G. Wang, T. Huang, Phys. Rev. D 89, 054019 (2014)

22. B. Aubert et al. [BaBar Collaboration], Phys. Rev. Lett. 90, 242001 (2003)

23. D. Besson et al. [CLEO Collaboration], Phys. Rev. D 68, 032002 (2003). [Erratum: Phys. Rev. D 75 , 119908 (2007)]

24. V.M. Abazov et al. [D0 Collaboration], Phys. Rev. Lett. 117, 022003 (2016)

25. The D0 Collaboration, D0 Note 6488-CONF (2016)

26. R. Aaij et al. [LHCb Collaboration], Phys. Rev. Lett. 117, 152003 (2016)

27. The CMS Collaboration, CMS PAS BPH-16-002 (2016)

28. J.R. Zhang, M.Q. Huang, Phys. Rev. D 80, 056004 (2009)

29. J.R. Zhang, M.Q. Huang, Commun. Theor. Phys. 54, 1075 (2010)

30. W. Chen, T.G. Steele, S.L. Zhu, Phys. Rev. D 89, 054037 (2014)

31. S. Zouzou, B. Silvestre-Brac, C. Gignoux, J.M. Richard, Z. Phys, C 30, 457 (1986)

32. B. Silvestre-Brac, C. Semay, Z. Phys, C 59, 457 (1993)

33. D. Ebert, R.N. Faustov, V.O. Galkin, W. Lucha, Phys. Rev. D 76, 114015 (2007)

34. Z.F. Sun, X. Liu, M. Nielsen, S.L. Zhu, Phys. Rev. D 85, 094008 (2012)

35. R.M. Albuquerque, X. Liu, M. Nielsen, Phys. Lett. B 718, 492 (2012)

36. S.S. Agaev, K. Azizi, H. Sundu, Phys. Rev. D 95, 034008 (2017)

37. M.A. Shifman, A.I. Vainshtein, V.I. Zhakharov, Nucl. Phys. B 147, 385 (1979)

38. V.M. Braun, A.V. Kolesnichenko, Phys. Lett. B 175, 485 (1986)

39. V.M. Braun, Y.M. Shabelski, Sov. J. Nucl. Phys. 50, 306 (1989). [Yad. Fiz. 50, 493 (1989)]

40. I.I. Balitsky, D. Diakonov, A.V. Yung, Phys. Lett. B 112, 71 (1982)

41. I.I. Balitsky, D. Diakonov, A.V. Yung, Z. Phys, C 33, 265 (1986)

42. J. Govaerts, L.J. Reinders, H.R. Rubinstein, J. Weyers, Nucl. Phys. B 258, 215 (1985)

43. J. Govaerts, L.J. Reinders, J. Weyers, Nucl. Phys. B 262, 575 (1985)

44. I.I. Balitsky, V.M. Braun, A.V. Kolesnichenko, Nucl. Phys. B 312, 509 (1989)

45. B.L. Ioffe, A.V. Smilga, Nucl. Phys. B 232, 109 (1984)

46. V.M. Belyaev, V.M. Braun, A. Khodjamirian, R. Rückl, Phys. Rev. D 51, 6177 (1995)

47. S.S. Agaev, K. Azizi, H. Sundu, Phys. Rev. D 93, 074002 (2016)

48. S.S. Agaev, K. Azizi, H. Sundu, Phys. Rev. D 93, 114007 (2016)

49. S.S. Agaev, K. Azizi, H. Sundu, Phys. Rev. D 93, 094006 (2016)

50. S.S. Agaev, K. Azizi, H. Sundu, Eur. Phys. J. Plus 131, 351 (2016)

51. L.J. Reinders, H. Rubinstein, S. Yazaki, Phys. Rep. 127, 1 (1985)

52. S.S. Agaev, K. Azizi, H. Sundu, Phys. Rev. D 93, 074024 (2016)

53. C. Patrignani, Chin. Phys. C 40, 100001 (2016)

54. P. Ball, V.M. Braun, A. Lenz, JHEP 0708, 090 (2007)

55. M.J. Baker, J. Bordes, C.A. Dominguez, J. Penarrocha, K. Schilcher, JHEP 1407, 032 (2014)

56. L. Maiani, F. Piccinini, A.D. Polosa, V. Riquer, Phys. Rev. D 89, 114010 (2014)

57. L. Maiani, A.D. Polosa, V. Riquer, Phys. Rev. D 94, 054026 (2016)

58. Z.G. Wang, Commun. Theor. Phys. 63, 325 (2015) 